This repurt was rrepaned as an sccount of work sponsored by an agency of the United States Government. Neither the United States Government nor any agency thereur, nor any of their employees, makes any warranty, express of implied, or essumes any legal liability or iesponsibilfty for the accuracy, completeness, or urefulness of any information, apparatus, product, or process disclosed, or refresents that ils use would not infringe privately owned rights. Reference herein to any specilic commercial product, process, or service by trade name, trademark. manufacturer, or otherwise does not necessarily constitute or imply its endorsenjent, recommerdalion, or favoring by the United Stetes Government or any agency thereof. The views United States Goremment w any agency therof not necessarily stale of reflect those of the

\title{
EQUILIBRIUM FLUCTUATION ENERGY OF GYROKINETIC PLASMA
}

\author{
John A. Krommes ${ }^{1}$ \\ Instittite for Theoretscal Physics, Unizersity of California \\ Santa Barblra, CA 98106 \\ W. W. Les \\ Plasma Physics Laboratory, Princeton University \\ P.O. Box 451, Princeton, Na:08544 \\ and \\ C. Oberman ${ }^{1,2}$ \\ Institute for Theoretical Physics, University of California \\ Santa Barbara, CA 99106
}

\begin{abstract}
A BSTRACT
The thermal equilibrium electric field fluctuation energy of the gyrolinetic model of magnetized plasma is computed, and found to be smaller than the well-known result $\langle\delta E \delta E\rangle(k) / 8 \pi=\frac{1}{2} T /\left[1+\left(k \lambda_{D}\right)^{2}\right]$ valid for arbitraily magnetized plasmas. It is shown that, in a certain sense, the equilibrium electric field energy is minimum in the gyrokinstic regime.
\end{abstract}

1 Permanent address: Plasma Physics Laboratary, Princeton University, P.O. Box 451, Princeton, NJ 08544.

2 Work partly performed at Physics Department, University of California, San Diego, La Joila, CA 92093. 


\section{INTRODUCTION: GYROKINETIC FLUCTUATION ENEPGY}

Although the particie simulation technique ${ }^{\mathfrak{1}}$ has been used for many years to study a wide variety of plasma phenomena, ${ }^{2}$ versinns based on the iull Lorentz equations of motion have met with only modest success when applied to problems of low frequency fluctuations in magnetized plasma-e.g., to drift waves in tok-maks. The difficulty lies with the fact that such codes resolve time scales which are much sharter than those of interest, and thus are unduly noisy and require substantial computation time. Recently, however, the picture bas changed: the systematic derivation of a nonlinear gyrokinetic equation ${ }^{3}$ in characteristic form ${ }^{4,5}$ has led to the advent of the gyrokinetic particle simulation technique ${ }^{6}$ Although many applications of this method remain to be made, the preliminary results ${ }^{7}$ are very encouraging: noise is very low, and the time steps may be relatively large. For the first time, it appears feasible to perform realistic simulations of nonlinea- low frequency phenomena in realistic geometry.

The principal application of the gyrokinetic simulation technique is to nonequilibrium phenomena. However, a well-established method (necessary but not sufficient) for testing the accuracy of such codes involves verifying that the code predicts the proper equilibrium electric field fluctuation spectrum $\varepsilon_{k} \doteq\langle\delta E \delta E\rangle(k) / 8 \pi$. (We shall use the notation "த" for "definition" and, later, " $\equiv "$ for "equivaleace"; the notation $\langle A B\rangle(k)$ means the Fourier transform with respect to $\rho$ of the translationally invariant two-point correlation ( $A(x+$ p) $B(\mathrm{x})\rangle$.) In unmagnetized or arbitrarily magnetized (in the sense of resolving the helical motion with an infinite number of harmo nics) equilibrium plasmas, $\varepsilon_{k}$ is well-known: it is predicted by the Euctuation-dissipation theorem (cf. Ref. 8 ) to be

$$
\varepsilon_{k}=\frac{T / 2}{l+\left(k \lambda_{D}\right)^{2}},
$$

a resuit independent of the magnetic field $B$. (Here $T$ is the temperature of a Gibbs ensemble, and $\lambda_{D} \doteq k_{D}^{-1}$, where $k_{D}^{2} \doteq k_{D_{e}}^{2}+k_{D_{i}}^{2}$ and $k_{D_{1}}^{2} \doteq 4 \pi\left(n e^{2}\right)_{s} / T$.) This resuit also holds for the guiding center plasma model..$^{9}$ However, it is interesting, and the source of a possible paradox., that (1) does not hold for the gyrokinetic pisma, where we find that the field energy is much smaller. The purpose of this paper is to discuss this issue. Indeed, we can show that, in a reasonable way, one can define a function $\varepsilon_{k}(B, n)$ which. as a function of $B$ and the density $n$, is smoothly connected to (1) and which exhibits a minimum in the gyrokinetic regime. This result is a persuasive argument in favor of the gyrckinetic method, as it implies that gyrokinetic simulations will be much quieter than simulations based on the full zquations of motion.

For definiteness, simplicity, and relevance to the extant gyrokinetic codes, we shall consider as the "exact" physical system to be studied a plasma with arbitrarily magnetized ions but with drift-kinetic electrons. (The extensions to the case of general electron magnetization are straightforward.) In thermal equilibrium. the linear dielectric function for 
this system may be written in the form

$$
\begin{aligned}
D(\mathbf{k}, \omega)=1 & +\left(\frac{k_{D}}{k}\right)^{2}+\left(\frac{k_{D e}}{k}\right)^{2}\left(\frac{\omega}{\sqrt{2}\left|k_{\mid i}\right| v_{e}}\right) Z\left(\frac{\omega}{\sqrt{2}\left|k_{\|}\right| v_{e}}\right) \\
& +\left(\frac{k_{D_{i}}}{k}\right)^{2}\left(\frac{\omega}{\sqrt{2}\left|k_{||}\right| v_{i}}\right) \sum_{n=-\infty}^{\infty} \Gamma_{n} Z\left(\frac{\omega-n \omega_{c i}}{\sqrt{2}\left|k_{\|}\right| v_{i}}\right) .
\end{aligned}
$$

Here $v_{s} \doteq\left(T / m_{s}\right)^{1 / 2}, Z(z)$ is the conventional plasma dispersion function, ${ }^{10}$ and $\Gamma_{n} \doteq$ $I_{n}(b) \exp (-b)$, with $b \doteq\left(k_{\perp} \rho_{i}\right)^{2}, \rho_{i} \doteq v_{i} / \omega_{c i}$, and $\omega_{c i} \doteq e B / m_{i} c$. (An alternative representation for the dielectric is discussed in App. A.)

The formula (2) is derived from the magnetized Vlasov equation. In general, however, we are not concerned exclusively with that complete expression, but rather with approximations to (2) appropriate to the physics regime we happen to be studying. The size of $\varepsilon_{k}$ then depends on the model; beuristically, we may predict its dependence on $B$ in the following way. Consider three models, which (loosely) correspond to various regimes of magnetic field or ion gyroradius: (a) the arbitrarily magnetized model, in which the helical motion of the ions is important and all Bessel harmonics must be retained in (2); (b) the gyrokinetic model, in which the fundamental sntity is the gyrocenter and only the $n=0$ harmonic is retained in (2)-that is,

$$
D_{g}(\mathbf{k}, \omega)=1+\left(\frac{k_{D}}{k}\right)^{2}+\sum_{s=e, i}\left(\frac{k_{D s}}{k}\right)^{2} \Gamma^{(s)}\left(\frac{\omega}{\sqrt{2}\left|k_{\|}\right| v_{s}}\right) Z\left(\frac{\omega}{\sqrt{2} \mid k_{\| !}^{\prime} v_{s}}\right)
$$

where $\Gamma^{(c)}=1$ and $\Gamma^{(i)}=\Gamma_{0}$; and (c) the guiding center model, the limit of model (b) in which $\rho_{i} \rightarrow 0$. In the arbitrarily magnetized model, which embraces the limit of unmagnetized ions (cf. App. A), high trequency, non-neutral fluctuations are present. and these lead to substantial noise- of the amount, in fact, described by Eq. (1). In the gyrokinetic model, valid for $|\omega| \ll \omega_{c i}$, the fast-moving ions have disappeared in favor of the slowly-moving gytocenters (whnge interaction with the electric field is described by $\Gamma_{0}$ ). These underge polarization drift, and, thus, tend to shield any fluctuations which might arise; the noise should therefore be smaller. In the guiding center regime, polarization drift is prohibited; both species move perpendicularly together at the $\mathbf{E} \times \mathbf{B}$ velocity. In the parallel direction, however, their speeds differ. Non-neutral Euctuations again arise. and lead to noise enhanced over the gyrokinetic value. In this sense of transitions between various models, we may thus expect a minimum of $\varepsilon_{k}(\boldsymbol{v})$ in the gyrokinetic regime.

Quantitatively, these results are a consequence of the fluctuation-dissipation theorem. which for plasma in the electrostatic approximation is

$$
\varepsilon_{k, \omega} \doteq \frac{1}{8 \pi}\langle\delta E \delta E\rangle(k, \omega)=-\frac{T}{\omega} \operatorname{Im}\left(\frac{1}{D(k, \omega)}-1\right)
$$


Equation (4) may be conveniently integrated over frequency ${ }^{8}$ by writing

$$
\begin{aligned}
\varepsilon_{k} \doteq \int_{-\infty}^{\infty} \frac{d \omega}{2 \pi} \varepsilon_{k, \omega} & =-\frac{T}{2 \pi} \int \frac{d \omega}{\omega} \operatorname{Im}\left(\frac{1}{D(\mathbf{k}, \omega)}-1\right) \\
& =-\frac{T}{2 \pi} \operatorname{Im} \mathrm{P} \int \frac{d \omega}{\omega}\left(\frac{1}{D(\mathbf{k}, \omega)}-i\right) \\
& =\frac{1}{2} T\left(\frac{1}{D(\mathbf{k}, \infty)}-\frac{1}{D(\mathbf{k}, \mathbf{0})}\right),
\end{aligned}
$$

where the last result was obtained by applying Cauchy's Theorem to the contour in Fig. 1. The result ( 1 ) follows from (5b) when one uses (2) to conclude that $D(k, 0)=1+\left(k \lambda_{D}\right)^{-2}$ and that $D(k, \infty)=1$ (the "vacuum" result).

Clearly (1) holds, independently of $B_{1}$ as long as all Bessel harmonics are retained in the dielectric - that. is, as long as we choose to describe the plasma completely, in terms of particles which obey the conventional Lorentz force law. (The time to approach equilibrium, which does depend on $B$, is not addreesed by this calculation.) In the gyrokinetic model, however, exact particle dynamics are replaced at the outset by gyrocenter dynamics, the dielectric tesponse for which is described by (3). (This is exactly true analytically, and true to an adequate degree of approximation in the explicit numerical gyrokinetic computational scheme. ${ }^{6}$ ) In this model, the general result (5b) still holds. However (dropping now the $\mathbf{k}$ dependence of $D$ for conciseness), the "vacuum" result $D_{g}(\infty)$ for the gyrokinetic plasma is not urity, but is rather

$$
\begin{aligned}
D_{g}(\infty) & =1+\frac{1}{\left(k \lambda_{D_{i}}\right)^{2}}\left(1-\Gamma_{0}\right) \\
& \approx 1+\left(\frac{k_{\perp}}{k}\right)^{2}\left(\frac{\omega_{p i}}{\omega_{c i}}\right)^{2} \quad\left(k_{\perp} \rho_{i} \ll 1\right) \\
& \approx 1+\left(\frac{\omega_{p i}}{\omega_{c i}}\right)^{2} \quad\left(k_{i l} / k_{\perp} \ll 1\right) .
\end{aligned}
$$

Of course, the last result, often somewhat imprecisely calle, the" dielectric for magnetized plasma, is well-known. ${ }^{11}$ However, its interpretation as a "vacuum" $(\omega \rightarrow \infty)$ dielectric has not always been clearly stated, especially with regard to its role in the fluctuationdissipation theorem. The point is that "vacuum" implies a consideration of the shortest time scales correctly resolved by the model. In the unmagnetized or arbitrarily magnetized case, those scales are arbitrarily short-horter, in particular, than the time for a spiraling ion (and, therefore, a collection of such ions) to respond to a perturbation at wave number k. Thus, for that case collective effects are not important $2 \mathrm{\alpha} \omega \rightarrow{ }^{*}$ ", and there $D(\infty)=1$. In the gyrokinetic case, however, the highest frequencies correctly resolved still satisfy $s \ll \omega_{c i}$, so that collective effects (involving the ion polarization drift) can operate even at $\nu=" \infty "$.

The result of all this is that for the gyrokinetic model

$$
\begin{gathered}
\varepsilon_{k}=\frac{1}{2} T\left(\frac{1}{1+\left(k \lambda_{D}\right)^{2}}-\frac{1-\Gamma_{D}}{\left(k \lambda_{D_{1}}\right)^{2}+1-\Gamma_{0}}\right) . \\
-4-
\end{gathered}
$$


which can be readily seen to be monotenic in $\Gamma_{0}$ (or $\rho_{i}$ ), with a maximum of amount (1) in the guiding center limit $\rho_{i} \rightarrow 0\left(\Gamma_{0} \rightarrow 1\right)$ and a minimum value of

$$
\varepsilon_{k, \min }=\frac{1}{2} T\left(\frac{1}{1+\left(k \lambda_{D}\right)^{2}}-\frac{1}{1+\left(k \lambda_{D_{i}}\right)^{2}}\right)
$$

in the limit of $k_{\perp \rho_{i}} \rightarrow \infty\left(\Gamma_{0} \rightarrow 0\right)$. It can also be viewed as the weak field limit $\rho_{i} \rightarrow \infty$ of the gyrokinetic model.

It is perhaps disquieting that the latter limit $\rho_{i} \rightarrow \infty$, apparently the unmagnetized limit, does not agree with result (1). There is really no intrinsic reason why it should; the gyrokinetic model is just that-a model-and need not be continuously connected to the unmagnetized limit. However, such continuity is conceptually useful to maintain. In the next section, we suggest a procedure or viewpoint whereby such a continuous connection can, in fact, be achieved.

\section{WHAT IS THE GYROKINETIC REGIME?}

In the previous section, we observed that the gyrokinetic plasma is described by a dielectric function which differs from that of the full plasma, and that equilibrium fuctuation theory can be applied straightforwardly to the gyrokinetic model. Now although that model can be studied in its own right, without reference to the properties of arbitrarily magnetized plasma, it is also of interest to discuss the relationship of the model to the full plasma dynamics. In this section, we shall define the gyrokinetic regime by describing a certain partition of the parameter space of density and magnetic field. We shall also give a procedure, based on frequency filtering, which enables one to view the equilibrium fluctuation properties of the gyrokinetic and other models as continuously connected to those of the arbitrarily magnetized or unmagnetized plasma. This procedure is primarily intended to be heuristic. We briefly discuss its relation to the more formal Hamiltonian method $^{5}$ in the next section.

As we have remarked, if cne begins with the full dielectric function (2) and computes $\varepsilon_{k}$ by integrating over all frequencies as in (5a), one will always recover the result (1). independent of magnetic field strength. Let us, however, consider insiead the frequencyfiltered function

$$
\varepsilon_{k}\left(\Omega ; \omega_{p i}, \omega_{c i}\right) \equiv \varepsilon_{k}(\Omega) \doteq \int_{-\Omega}^{\Omega} \frac{d \omega}{2 \pi} \varepsilon_{k, \omega},
$$

where $\Omega$ is a fixed cutoff and where $\varepsilon_{k, w}$ is computed using the full dielectric (2). (Practically, ii would be desirable to use a low-pass filter smoother than the box fiter implied by (9). However, the following heuristic arguments are independent of such details.) ts s'pr and $\omega_{c i}$ are continuously varied, $\varepsilon_{k}(\Omega)$ will continuously vary, with a value which may be thought of as arising from an approximate dielectric or model appropriate to the frequency range $|\omega|<\Omega$ and the chosen values of $\omega_{p i}$ and $\omega_{c i}$. If, for example, $\Omega \ll \omega_{c 1}$. then one is 
entitled to replace $D(\nu)$ in $(5 a)$ by the approximate expression appropriate to $\omega_{i} \ll \mu_{c 1}$. Haying done this on the real axis, one may, if he chooses, analytically continue the approximate dielectric into the complex, plane in order to expedite evaluation of (5a). (Contcur integration is not required, of course. If the bulk of the power lies in weakly damped normal modes, one may wish to perform the integration in (9) directly by integrating over the resonances.) The resulting function of $\omega$ defines the dielectric model appropriate to the chosen parameter regime. For the cited example, one obtains to lowest order in $\Omega / \mathrm{sici}_{c i}$ the gyrokinetic dielectric (3).

In Fig. 2, we consider the parameter space of $\left(\omega_{p i}, \omega_{c i}\right)$ at fixed $k_{\|}, k_{\perp}$, and $\Omega$. The fundamental lines $\omega_{p i}=\Omega, \omega_{c i}=\Omega$, and $\omega_{c i}=\omega_{p i}$ divide the space into six regimes. When the line $k_{\perp} \rho_{i}=1$, which separates the unmagnetized from the magnetized regimes, is added, three more subdivisions appear. (One is not shown in Fig. 2.) The regimes $k_{\perp} \rho_{i} \gg 1$ (e.g., I and II) define the unmagnetized regime, where the ion susceptibility (2) is essentially equivalent to the unmagnetized dielectric. (For a brief review of that limitiag procedure, see App. A.) The normal modes in this regime are modified Langmuir oscillations:

$$
\omega_{L}^{2} \approx \omega_{p i}^{2}+\left(k_{\|} / k\right)^{2} \omega_{p e}^{2} .
$$

(See App. B for a brief discussion of the equilibrium normal modes of the present system.) In Fig. 2, the approximate orderings of such normal modes are identifed by vertical arrows pointing to their approximate position in the inequalities which define the regimes. When those modes are of frequency higher than $\Omega$, they are enclosed in parentheses and indicated with dashed arrows in order to remind the reader that those resonances would not be counted during the integration of (9) on the real axis. In regimes III-VIII, the normal modes are the lower hybrid oscillations, the dispersion relation of which is

$$
\omega_{l h}^{2} \approx \omega_{c i}^{2}+\left(\frac{k_{\perp}^{2}}{k^{2}}\right) \omega_{p i}^{2}+\left(\frac{k_{\|}^{2}}{k^{2}}\right) \omega_{p e}^{2}
$$

and a lower frequency mode

or

$$
\omega^{2} \approx \frac{k_{\|}^{2} \omega_{p e}^{2} / k^{2}}{1+\omega_{p i}^{2} / \omega_{c i}^{2}} \quad\left(k_{\|} \ll k\right),
$$

$$
\begin{aligned}
& \omega_{A} \approx \pm\left(\frac{k_{\|}}{k}\right)\left(\frac{m_{i}}{m_{e}}\right)^{1 / 2} \omega_{c i} \quad\left(\omega_{p i} \gg \omega_{c i}\right), \\
& \omega_{L d} \approx \pm\left(\frac{k_{\|}}{k}\right) \omega_{p e} \quad\left(\omega_{p i} \ll \omega_{c i}\right),
\end{aligned}
$$

where $\omega_{A}$ is an electrostatic version of the shear Alfven wave and $\omega_{L d}$ is the modified Langmuir wave in the drift-kinetic limit. It is importart to note that $\omega_{A}$ is intrinsically quasineutral due to the shielding effects of the ion polarization drift whereas $\omega_{L}, \omega_{l h}$, and $\mathrm{A}_{L 1}$ are non-neutral oscillations. The gyrokinetic regime is $V$, which can be reached. for 
example. by tracing a patb from the origin through regimes I, II, and IV, or equivalently through III and IV. In the gyrokinetic regime, $w_{\text {Ih }}$ is large compared to $\Omega$, so only the $\therefore$.A modes contribute to the frequeacy-filtered $\mathcal{E}_{k}(\Omega)$. In nonequilibrium situations. the drift wave would exist in this regine and dominate the noise spectrum; however, that waye is absent in the homogeneous equilibrium limit. Thus, it should now be clear why equilibrium noise is substantially lower in the gyrokinetic regime than in the unmagnetized limit. Notice that the poise remains low if the path goes to $\infty$ in regime $V$; however, if the path goes to regimes VI and VII, the presence of $\omega_{L d}$ will raise the noise back to its unmagnetized value.

\section{DISCUSSION AND CONCLUSIONS}

The heuristic frequency filtering scheme introduced in Sec. II provides a successful generalization of the well-known result (1). However, there is a substantial amount of arbitrarimess in (9). Filters other than (9) can obviously be devised which would behave differently. However, at least in the low frequency regine V-VII, there is a much more profound procedure for effecting such frequency filtering which, in fact, provided the motivation for the choice (9)-namely, the Hamiltonian-Lie method of symplectic transformations. In this approach, which we have explored in a previous article, ${ }^{5}$ high frequency dynamics are not arbitrarily neglected or filtered away; rather, they are merely segregated into the generating function of a symplectic transformation. A Hamiltonian and a (gyro-) kinetic equation for gyrocenters can be defined by systematically transforming away gyro-angle dependence order by order. This averaging transformation, which physically removes the bigh frequency motions from the gyrokinetic Hamiltonian and kinetic equation. is the formal procedure which effects the frequency filtering introduced heuristically in the last section. The consistency of the two approaches is demonstrated when one notes that the linear response theory of that system leads precisely to (3). Furthermore, the Hamiltonian nature of this problem Ieads one naturally to a numerical scheme to simulate the gyrocenter dynamics; of necessity, the equilibrium fluctuation properties of that simulation plasma must agree with (T). [A similar procedure applicable to the high frequency regime (II and IV) has yet to be developed.]

Thus, we may summarize our results as follows. We have computed ihe equilibrium electric field energy for gyrokinetic plasma by a careful application of the fluctuationdissipation theorem. The low level of equilibrium noise in that system lends credence to the validity of the gyrokinetic simulation approach. Finally, we have suggested a simple scheme by which we can understand the relation of the gyrokinetic model to the arbitrarily magnetized plasma.

\section{ACKNOWLEDGMENTS}

This work -vas supported in part by U.S. D.o.E. Contract Yo. DE-AC02-76-CHO3073, and in part by the National Science Foundation under Grant Yo. PHY82-17853. 
supplemented by funds from the National Aeronautics and Space Administration.

\section{APPENDDX A: THE UNMAGNETIZED LIMIT}

Here, we remind the reader of the steps necessary to establish the colnection between the arbitrariiy magnetized susceptibility (2) and the unmagnetized susceptibility. There are at least two terbniques of interest.

In the first method, sast closely related to the representation (2), we need to show that

$$
\begin{aligned}
I & \doteq \sum_{n=-\infty}^{\infty} \Gamma_{n} \int d v_{\|} \frac{f_{M}\left(v_{\|}\right)}{\omega-k_{\|} v_{\|}-n w_{\mathrm{ci}}} \\
& \rightarrow \int d \mathbf{v} \frac{f_{M}(\mathbf{v})}{\omega-\mathbf{k} \cdot \mathbf{v}}
\end{aligned}
$$

as $k_{\perp \rho} \rightarrow \infty$. We may use the asymptotic form

$$
\Gamma_{n}(b) \sim\left(2 \pi b^{2}\right)^{-1 / 2} \exp \left(-n^{2} / 2 b^{2}\right)
$$

and have, upon introducing the variable $u \doteq n \Omega / k_{\perp}$,

$$
I \approx \int d u\left(2 \pi v_{t}^{2}\right)^{-1 / 2} \exp \left(-\frac{u^{2}}{2 v_{t}^{2}}\right) \int d v_{\|} \frac{f_{M}\left(v_{\|}\right)}{\omega-k_{\|} v_{\|}-k_{\perp} u} .
$$

If we choose $\mathbf{k}_{\perp}$ to be in the $\mathbf{x}$ direction for definiteness, we may interpret is as $v_{x}$, and $k_{\left\|,{ }^{r}\right\|}+k_{\perp} u=k_{\| \mid} v_{\|}+k_{\perp} \cdot v_{\perp}=\mathbf{k} \cdot \mathbf{v}$. The result (A2) then follows upon multip'ying (A4) by $1=\int d v_{\|} f_{M}\left(v_{\|}\right)$.

The above technique is not rigorous because the uniform validity of the as: "ptotic form is questionable. A sounder procedure, which leads to the same result and , ...ch is particularly well suited for representing limiting situations, is to derive an integral representation ${ }^{12}$ for the ion susceptibility:

$$
\begin{aligned}
x_{i}(\mathbf{k}, \omega)=1 & +\frac{k_{D}^{2}}{k^{2}}-\frac{k_{D}^{2}}{k^{2}}(i \omega) \\
& \times \int_{0}^{\infty} d \tau \exp \left(i \omega \tau-\frac{k_{1}^{2} v_{t}^{2}}{\omega_{c}^{2}}\left[1-\cos \left(\omega_{c} \tau\right) !-\frac{1}{2} k_{i}^{2} v_{t}^{2} \tau^{2}\right)\right.
\end{aligned}
$$

From (AS), the limits $\omega_{c} \rightarrow 0$ or $w \rightarrow 0$ follow immediately. 


\section{APPENDIX B: EQUILIBRIUM NORMAL MODES}

The model considered in the main body of the paper is slightly unusual because the electrons and ions were not treated symmetrically: the electrons were drift-kinetic while the ion magnetization was arbitrary. Here we comment very briefly on the thermal equilibrium normal modes for that system.

First, consider the limit of completely unmagnetized ions. In this case, the dielectric retains the form (2), but with the ion susceptibility replaced by

$$
\left(\frac{k_{D_{i}}}{k}\right)^{2}\left(\frac{\omega}{\sqrt{2}|k| v_{i}}\right) Z\left(\frac{\omega}{\sqrt{2}|k| v_{i}}\right) \text {. }
$$

In general, such a dielectric would support an ion sound mode in the frequency range $k v_{i}<\omega<k_{\|} v_{e}$. However, in thermal equilibrium such a fluctuation is heavily Landat damped and does not qualify as a normal mode. Langmuir oscillations persist, however. Upon expanding in the fuid limit $\omega>k v_{i}, \omega>k_{\|} v_{e}$, one is readily led to the form (10).

For the magnetized case, we shall restrict our attention to the cold limit. It is convenient to begin with the well-known dispersion relation for arbitrary electron magnetization, ${ }^{13}$

$$
0=k_{\perp}^{2}\left(1-\sum_{s} \frac{\omega_{p s}^{2}}{\omega^{2}-\omega_{c s}^{2}}\right)+k_{\|}^{2}\left(1-\sum_{s} \frac{\omega_{p s}^{2}}{\omega^{2}}\right)
$$

which simplifes in the drift-kinetic limir $\omega_{c e} \rightarrow \infty$ to

$$
0=k_{\perp}^{2}\left(1-\frac{\omega_{p i}^{2}}{\omega^{2}-\omega_{c i}^{2}}\right)+k_{\|}^{2}\left(1-\frac{\omega_{p}^{2}}{\omega^{2}}\right)
$$

where $\omega_{p}^{2}=\omega_{p e}^{2}+\omega_{p i}^{2}$. In the bigh frequency limit $\omega>\omega_{c i}$, (B.3) supports the normal mode (11), which is just the lower hybrid oscillation. The conventional dielectric factor $\omega_{p e}^{2} / \omega_{c e}^{2}$, which describes the electron polarization drift, vanishes in the drift-kinetic limit being considered here.

In the low frequency limit $w<\omega_{c i}$, one finds from (B3) the normal node (12).

\section{References}

'J. M. Dawson, Phys. Fluids 10, 2 (1971).

${ }^{2}$ See, for example, J. M. Dawson, H. Okuda, and B. Rosen, in Methods of Computational Physics (Academic Press, Yew York, 197€), Vol. 16, p. 281. 
"E. A. Frieman and L. Chen, Princeton Plasma Physics Laboratory Report vo. PPPL1834. I981.

'W. W. Lee, Phys. Fluids 26, 556 (1983).

${ }^{5}$ D. H. E. Dubin, J. A. Krommes, C. Oberman, and W. W. Lee, Phys. Fluids 26, 3524 (1983).

${ }^{6}$ W. W. Lee, Princeton Plasma Physics Laboratory Report No. PPPL-2077, 1985.

${ }^{7}$ W. W. Lee, J. A. Krommes, C. Oberman, and R. A. Smith, Phys. Fluids 27, 2652 (1983).

${ }^{8}$ S. Ichimaru, Basic Principles of Plasma Physics--A Statistical Approach (Benjamin. Reading, Massachusetts, 1973).

${ }^{9}$ J. B. Taylor and B. McNamara, Phys. Fluids 14, 1492 (I971).

${ }^{10}$ B. D. Fried and S. D. Conte, The Plasma Dispersion Function (Academic, New York. 1961).

${ }^{11}$ S. Chandrasekhar, Plasma Physics (University of Chicago Press, Chicago, 1960).

${ }^{12}$ I. Lornstein, Phys. Rev. 109, 10 (1958).

${ }^{13}$ T. H. Stix, The Theory of Plasma Waves (McGraw-Hill, New York, 1962). 


\section{FIGURE CAPTIONS}

Fig. 1. The integration contour used to deduce Eq. (5b): $\int_{A}=-\int_{B}-\int_{C}$.

Fig. 2. Parameter space for thermal equilibrium plasma, with drift-kinetic electrons and arbitrarily magnetized ions, as a function of magnetic field $\left(\omega_{c i}\right)$ and density $\left(\omega_{p 1}\right)$. See Sec. II for detailed explanation. 


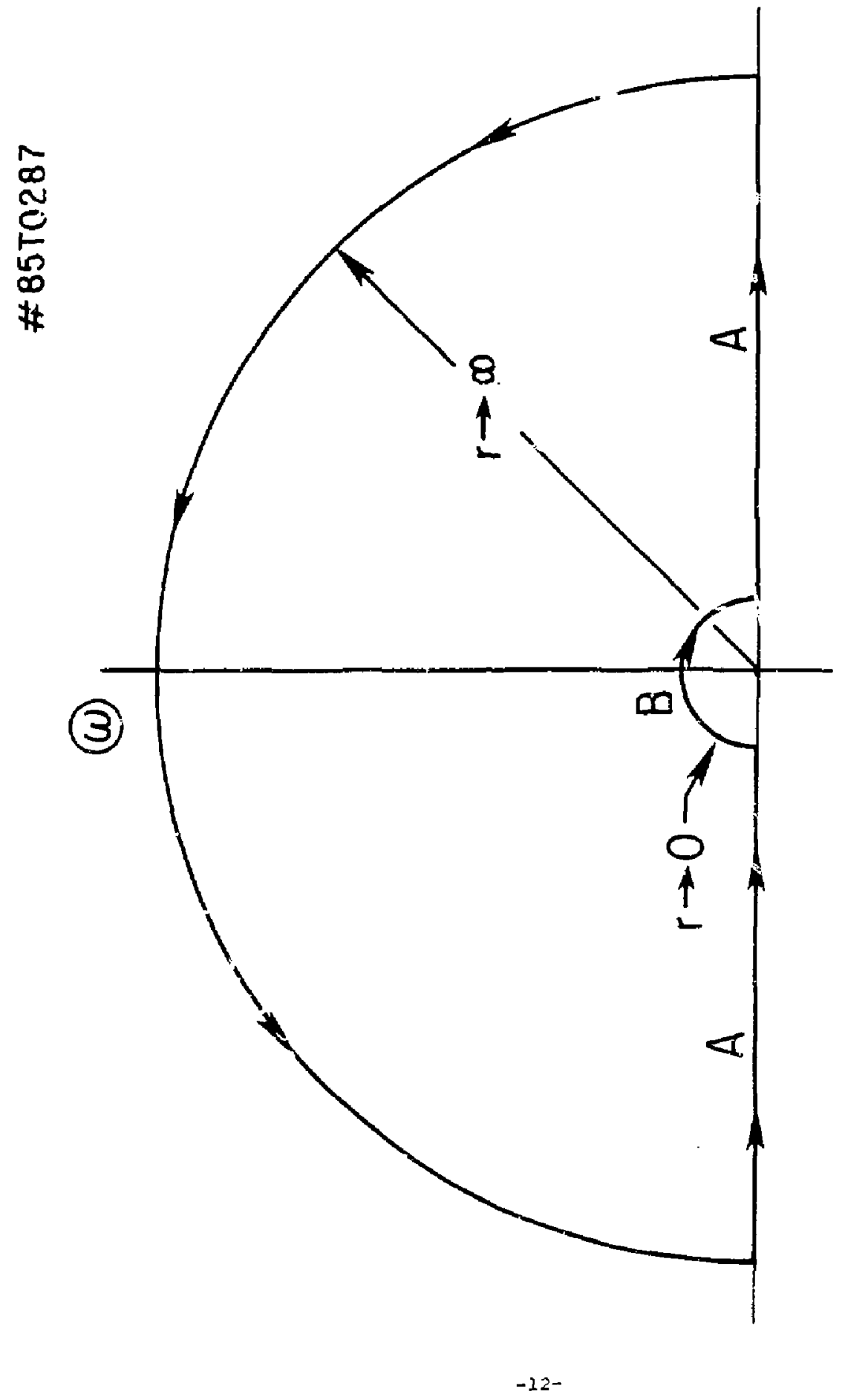

- 


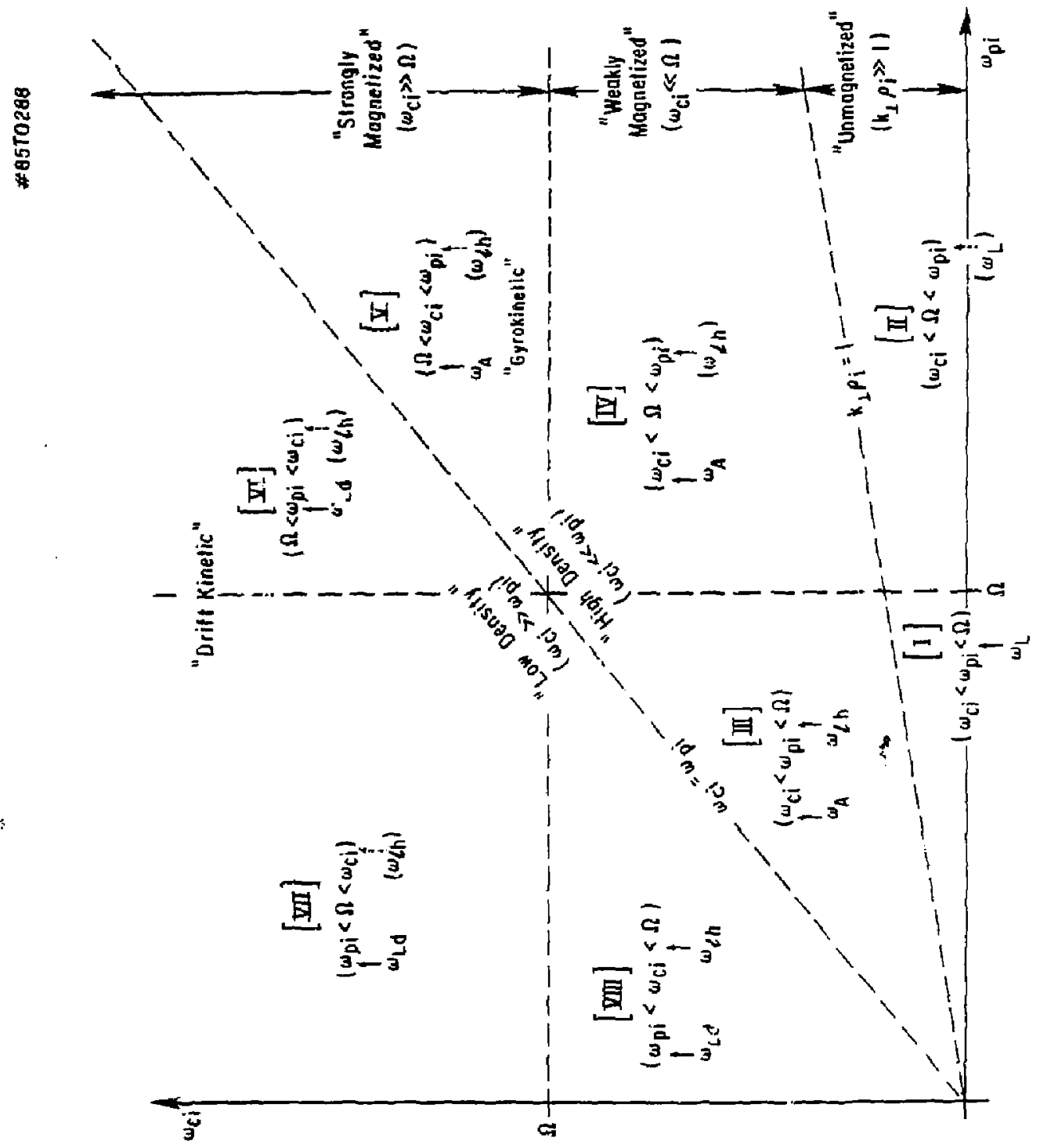


Plasing kes Lab, Austra Mac'l Univ, AUsiRat.tA x. Eran' J. Pagloni, thiv of wollongong, ALSTRALIA exof. I.R. Jones, Flinders thivt, ALSIRALIA prof. M.H. Erennal, thiv Sytrey, ausIRALTA Prof. E. Cag, Inst Theo Arys, Alsisua Prof. Frark thertest, Inst thoocetische, BEIGtuM Dr. D. Falumbs, og XII Futsion Ptog, BesGIIM Bcole Royale tilitaire, Lab de Fiys riasmas, BEIGIM Dr. P.M. Sakanaka, Univ Estadual, Exartl D. C.R. Janes, thiv of Alberta, CMAPA Prof. J. Tetchiman, Ujier of ventreal, OARDA Dr. H.M. Scarsgard, thiv of Saskatctrewan, CANADA Prot. S.R. Steenivasan, thiversity of Calgary, GapaA Prof. Mudor w. Joinston, INRS-thergie, CARDA or. Hannes Garmand, Uhiv BAtish Columbia, GANDs

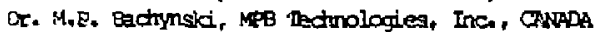
Chalk River, Nucl Iab, CARna

Thengun Ii, SN Inst Physics, CHIMA

Library, Ising tha Uniwersity, Grm

Librarian, Institate of Physics, CHMNa Inst Plasua Phys, Acaderia 5inica, Cruth nT. Peter Lukac, Kamenskelo thiv, cancHostovarta The Librarian, Oxthrom Laboratory, Exrast irof. Bchataman, Observatoire de NAce, FrancE J. REcjet, COHEPG, FFANCE AM Dupos Library, AM DUPas Library, EPANCE Dr. Irom Mal, Acadeny Bibliographic, HDK KDTS Preprint Iibrary, Cent Pea Inst Ehys, IJWOPY Dr. R+K. Ahajlani, Wikran Univ. IrUIA or. 3. Dasqupta, Saha Inst, IDDIA Dr. P. Kow, Fhricical Reocarch Lab, HOA

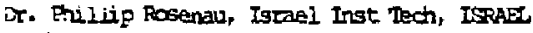
Ecot.. S. Guperman, Tel Aviv iniluersity, ISFAEL ProE. G. Rostagni, thiv Di Paciov, IMTLY Iibratian, Inc'l Cra theo Enys, IITLY

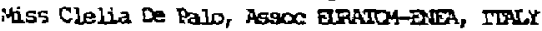
aiblioteca, del OR ERATIOs, ITLYY

DT. H. Yamato, Thahiha Pes \& Dev, JAPAN

Direc. Dept, ig. Tokamik Dev, JABR, JARAN

Erof. Mbluyuki Inase, Uhiversity of Tokyo, JAPAN Nesearch Info Center, Nagoya thiversit/, JAPAN

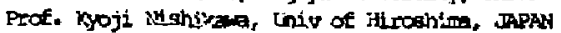
Prof. Sigera MGCi, JARRI, JAPAN

Prof. S. Ranaka, Ryoto Chiversity, IAPAN ilizary, Kyoto thiversity, JAPAs Prof. Ichiro Kavabani, Niten thiv, IAPAN Prot. Satoshi Itoh, Kyssish University, JAPAV Dr. D.I. Goi, Adv. Inst Sci \& Tech, KORGA Tech Info Division, KAPRI, KCREA. aibliothede, Fom-Inst Voor Plasma, NELHERLANDS

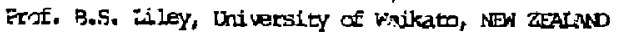
Prof. J.A.C. Cabral, Irit Superice Teon, Forducal
Dr. Octavian Petrus, ALI CULA University, ROMANIA Prof. M.A. Heliberg, Unilessity of Malal, $\$$ AftuCA DE. Johan te villiers, Plasma Physics, Nucor, 50 AFrteA Fusion Div. Iilmary, JEN, SPATI

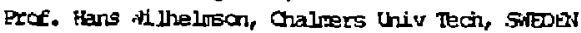

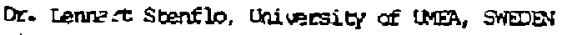
Lithary, Royal jist Tech, SWDODN

Centre o' Recherchesen, Exole Polyrect Eed, SwTLERLAw

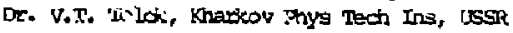
Dr. D.D. Rjutov, Siberian Acad Sci, USSR D. G.Ar Eliseev, Murchatoy Instiutte, USSR Dr. V.A. Glukhikh, Inst Electro-Fhysical, USSR Instidte Gen, Thysics, ISSR

Prof. T.J.M. Boyd, Univ College is waleg, HALES

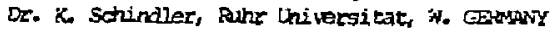
Nuclear pes Estab, vilion Ltd, w. greyant inbrarian Maxtlandk Instibut, \%. GDRENy Bibliothek, Inst Plasmuforsctung, W. GDWANY Erof. R.x. Janev, Inst Ehys, rugustavia

\author{
REPRODUCED FROM \\ BEST AVAILALLE COPY
}

